\section{Modelo não linear de localização de instalações de serviços de saúde com indicador de acessibilidade incorporado}

\author{
A nonlinear model for localization of hospital \\ services as an indicator of accessibility
}

\section{Modelo no lineal de localización de instalaciones de servicios de salud con indicador de accesibilidad incorporado}

Silviana Cirino 1,2

Louis Augusto Gonçalves 2

Mirian Buss Gonçalves 2

Eduardo Souza de Cursi 3

doi: 10.1590/0102-311X00185615

\section{Resumo}

Este trabalho propõe uma abordagem diferenciada para a localização de unidades prestadoras de serviço público. Diferentemente de métodos que se atêm unicamente à eficiência locacional para a distribuição de unidades prestadoras de serviços públicos, apresenta-se um modelo não linear que incorpora um indicador de acessibilidade em seu bojo, que permite rejeitar soluções cuja acessibilidade esteja fora de parâmetros mínimos arbitrados como aceitáveis. O método visa a minimizar o tempo total gasto pela população de uma região para alcançar uma rede de prestação de serviços públicos, todavia, controla a amplitude entre a região com maior e menor acessibilidade à rede de prestação de serviços. A solução que se obtém não é tão eficiente, quando comparada a outros modelos como p-medianas, no tocante ao custo total despendido pela população como um todo para ter acesso ao sistema, entretanto, busca evitar que áreas mais afastadas tenham dificuldades amplificadas em virtude da desproporção de tempos de viagem necessários. O modelo foi testado em uma região da rede hospitalar do Estado de Santa Catarina, Brasil, e os resultados mostram que a incorporação do indicador aponta para uma melhoria em relação à conjuntura atual da distribuição de unidades hospitalares. O uso da metodologia sugerida pode ser útil, como uma ferramenta que contribui com subsídios, para a elaboração de um planejamento que permita uma alocação equilibrada de recursos, quando da instalação de unidades prestadoras de serviços à população.

Dinâmica não Linear; Acesso aos Serviços de Saúde; Serviços de Saúde; Planejamento em Saúde

\author{
Correspondência \\ S. Cirino \\ Instituto Federal de Educação, Ciência e Tecnologia de \\ Santa Catarina. \\ Rua José Lino Kretzer 608, Campus São José, São José, SC \\ 88103-310, Brasil. \\ silviana_cirino@yahoo.com.br \\ 1 Instituto Federal de Educação, Ciência e Tecnologia de Santa \\ Catarina, São José, Brasil. \\ 2 Universidade Federal de Santa Catarina, Florianópolis, Brasil. \\ 3 Institut National des Sciences Appliquées de Rouen, Saint \\ Etienne du Rouvray, France.
}




\section{Introdução}

A moderna gerência de recursos públicos exige eficiência máxima na prestação de serviços estatais. Diferentemente da iniciativa privada, que visa a maximizar lucros, no sistema público almeja-se maximizar o acesso do serviço à população, otimizando a utilização dos recursos disponíveis. Para isso, é preciso reduzir o custo que a população despende para alcançar uma unidade prestadora de serviço, o que não deve ser realizado de forma imponderada. Com o intuito de fomentar a equidade na utilização do serviço, precisa-se buscar uma forma em que todas as localidades tenham acesso ao sistema, evitando que uma determinada região fique isolada de tal forma que equivalha na prática à ausência do serviço para seus habitantes 1 .

Modelos matemáticos clássicos de localização são eficientes quanto à economia gerada para a sociedade, todavia, desprezam o empenho para os habitantes de uma localidade específica, geralmente distante dos centros e com baixa densidade demográfica, para alcançar um sistema de prestação de serviços públicos. Nesse sentido, torna-se importante ter em mãos um indicador, já incorporado ao modelo, que seja capaz de mensurar o esforço necessário para os habitantes terem acesso ao serviço, e que torne possível gerar soluções em que este esforço seja um pouco melhor distribuído, de forma a não comprometer o uso pela população mais vulnerável em razão da distância em que se encontra. Dessa forma, modelos capazes de encurtar o desequilíbrio para acesso ao sistema, mas ao mesmo tempo o mantendo viável, sem alterar de forma desarrazoada o custo para a sociedade, são normalmente bem-vindos tanto pelos gestores governamentais quanto pela população 2 .

No presente estudo, limita-se o conceito de acessibilidade, restringindo-o como o tempo necessário para alcançar uma instalação prestadora de serviços a partir de uma localidade, o que na literatura é conhecido como acessibilidade geográfica 1,2,3,4,5,6,7. O conceito de equidade também é restringido, de forma a considerar duas regiões equitativas se o esforço feito pelos usuários para terem acesso a uma unidade médica é similar, esforço neste caso medido em tempo de deslocamento.

Grande parte dos textos disponíveis 4,8,9 trata da avaliação de acessibilidade de um certo cenário, seja real ou simulado, encontrando a solução ótima segundo um determinado modelo. A seguir compara o resultado obtido pelo modelo, com os dados reais, para tirar conclusões sobre a qualidade da distribuição 4,9. Em suma, a localização é determinada a priori e a acessibilidade é verificada a posteriori. Ao contrário, no presente trabalho, propõe-se um modelo que incorpora um indicador que delineia as soluções geradas a um limite arbitrário mínimo de acessibilidade, já durante os cálculos. Isso permite obter soluções com níveis mínimos de acessibilidade e ao mesmo tempo com grau de ineficiência baixo quando comparadas a soluções que a desconsideram em seu bojo. O estudo, portanto, tem como objetivo aplicar um modelo capaz de gerar uma distribuição de unidades de saúde que tenha custo baixo para ser acessado, com um mínimo estipulado de equidade entre as localidades. Os resultados obtidos podem ser utilizados como ferramentas de apoio à decisão para aprimorar a situação de uma determinada região, em relação à prestação de serviços de saúde.

\section{Método}

Modelos matemáticos de localização buscam soluções que satisfaçam premissas previamente estabelecidas em seu conjunto de restrições. Um dos modelos mais usados para localizar um número fixo de unidades prestadoras de serviços públicos em uma região é o de p-medianas, que minimiza o custo que os residentes, de uma região discretizada, consomem para alcançar uma rede de unidades, como hospitais ou escolas 4 . Não se avalia, no modelo de p-medianas, o esforço necessário para que um grupo específico tenha acesso a uma determinada unidade de prestação de serviços. Isso possivelmente causa um desequilíbrio, pois, apesar de se encontrar a distribuição mais econômica de unidades prestadoras para a população como um todo, populações mais afastadas podem ficar praticamente impedidas de obter acesso ao sistema. Todavia, inserir indicadores como restrição em métodos lineares, como p-medianas, é extremamente custoso (ou mesmo inviável) do ponto de vista computacional, de forma que uma opção mais tangível é aplicar métodos não determinísticos, como algoritmos genéticos 10 e de otimização não linear. 
Uma investigação que considere todas as variáveis possíveis que possam determinar o quanto um certo grupo tem acesso a um serviço público pode ser demasiadamente complexo e até subjetivo, visto que pessoas idosas, deficientes físicos, moradores carentes, ou qualquer outro grupo em condição de vulnerabilidade social, precisam de um sistema adaptado às suas necessidades para que o serviço prestado lhes seja acessível 11. No entanto, com o intuito de restringir variáveis determinantes, limitou-se, neste trabalho, à dispersão espacial de unidades hospitalares e à densidade demográfica de uma região como os elementos que representam a acessibilidade aos serviços de saúde, fato que é corroborado por autores renomados 5,12. Nesse contexto, considera-se que somente o tempo esperado de viagem entre uma localidade e o hospital mais próximo, e a população nela residente, representam o esforço para o acesso à saúde do universo de pessoas que faz uso do serviço. O tempo de viagem foi tido como preferível à distância por levar em conta a qualidade da via e possíveis obstáculos naturais entre os nós. Em resumo, o estudo, apesar de desconsiderar necessidades especiais que um determinado indivíduo ou grupo minoritário possua, visa a evitar que populações em regiões mais distantes de centros tenham esta desvantagem ainda mais ampliada.

Considerando os fatores descritos, propõe-se um modelo de localização cuja função objetiva seja minimizar o tempo médio de viagem gasto pelos usuários para alcançar as unidades médicas. A cada iteração do algoritmo uma solução aleatória é gerada e vai passo a passo sendo melhorada, e é penalizada se não estiver satisfazendo um limite mínimo arbitrado de acessibilidade.

O indicador de acessibilidade da localidade $i$, simbolizado por $A_{i}$, considerado neste artigo é apresentado a seguir.

Seja:

$A_{i}=$ acessibilidade da zona $i$;

$A t r_{j}=$ atratividade da zona $j$;

$c_{i j}=$ distância (ou tempo de viagem) entre $i$ e $j$;

$\gamma=$ parâmetro;

$n a=$ número de unidades hospitalares consideradas no cálculo do indicador.

$$
A_{i}=\frac{\sum_{j=1}^{n a} \operatorname{Atr}_{j} \exp \left(-y c_{i j}\right)}{\sum_{j=1}^{n a} A t r_{j}}
$$

Com base na medida da acessibilidade obtida, buscou-se limitá-la a valores máximo e mínimo aceitáveis e, por fim, criar um parâmetro percentual que reflita a diferença de acessibilidade entre os extremos. Nesse contexto é definido o parâmetro $\beta$ :

$$
\frac{\operatorname{Max} A_{i}-\operatorname{Min} A_{i}}{\operatorname{Max} A_{i}} \leq \beta
$$

Em que:

$\operatorname{Max} A_{i}=$ valor máximo da acessibilidade;

$\operatorname{Min}_{i}=$ valor mínimo da acessibilidade;

$\beta=$ variação percentual de acessibilidade.

Para mensurar a atratividade, limitou-se a usar apenas o número de leitos como medida. Para as zonas que têm leitos hospitalares disponíveis, esse número, conforme os dados do Cadastro Nacional de Estabelecimentos de Saúde (http://cnes.datasus.gov.br, acessado em 08/Jul/2014), foi utilizado. Para as zonas que não contam com hospitais, foram supostos dois leitos para cada mil habitantes.

$\mathrm{O}$ indicador de acessibilidade proposto é classificado como gravitacional 5,6,10,13,14,15, já que incorpora a separação espacial entre os pares origem e destino e uma medida de atratividade. Nesse tipo de indicador, quanto maior a distância (ou tempo de viagem), menor a acessibilidade; quanto maior a atratividade, maior a acessibilidade. É um indicador "crescente", ou seja, quanto maior o valor obtido para o indicador, melhor é a acessibilidade. Em relação ao parâmetro $\beta$, que mede a variação percentual de acessibilidade, quanto mais próximo de zero o parâmetro estiver, mais equitativa será a distribuição. 
O modelo de localização de instalações, preliminarmente proposto em Cirino et al. 16 e aperfeiçoado conforme apresentado a seguir, tem por objetivo minimizar a distância total ponderada percorrida (ou tempo de viagem), genericamente dado por:

Minimizar

$$
f(x)=\sum_{j=1}^{n} \lambda(I(x, j), j)+\left(\beta_{j}-\beta\right) . \text { Pen }
$$

Sujeito a

$$
\begin{gathered}
\sum_{i=1}^{n} x_{i}=k \\
\mathrm{x}_{\mathrm{i}} \in\{0,1\} ; 1 \leq \mathrm{i} \leq \mathrm{n}
\end{gathered}
$$

Em que $k$ é um número inteiro e positivo de instalações que se deseja localizar; $x=\left(x_{i}: 1 \leq i \leq n\right)$ é um vetor de incógnitas no qual $x_{i}=1$ se houver instalação em $i$ e $x_{i}=0$ caso contrário; $n$ é o número de nós considerados no problema; $C$ : $\{1, \ldots, n\} ; I(x, j)=\arg \min \left\{x_{i} \alpha(i, j): 1 \leq i \leq n\right\}$ corresponde ao índice $i$ tal que $x_{i}=1$ e $\alpha(i, j)$ é mínimo; $\alpha(i, j)$ é gerada por meio da matriz de distância (ou tempo de viagem) $\left(d_{i j}\right)_{n} ; \lambda(i, j)$ é gerada usando-se a matriz de distâncias (ou tempo de viagem) $\left(d_{i j}\right)_{n}$ e da matriz de pesos $w$. A função objetivo é penalizada pela adição da multiplicação entre a variação do valor do parâmetro $\beta_{j}$ encontrado na iteração e do valor de $\beta$ arbitrado e Pen, um número grande que causa relevância ao valor da função objetivo. No experimento arbitrou-se $P e n=10^{6}$.

A solução é representada por:

$$
x^{*}=\arg \min \left(f(x): \sum_{i=1}^{n} x_{i}=k\right) .
$$

Para gerar $\alpha$ considera-se uma função $\varphi:[0,+\infty[\rightarrow R$ estritamente negativa e estritamente crescente e $\alpha(i, j)=\varphi\left(d_{i j}\right)$. Nesse caso, $I(x, j)$ corresponde ao índice $i$ tal que $x_{i}=1$ e $d_{i j}$ é mínimo. Usualmente $d_{i j}=d_{j i}$ e $d_{i i}=0$, mas o método se aplica quando estas condições não estão satisfeitas. A função usada foi $\varphi(z)=-1 /(z+\eta), \eta>0 . \alpha: C x C \rightarrow R$ é uma função estritamente negativa; quanto menor $\alpha(i, j)$, mais chances haverá de alocar a zona $j$ ao hospital $i$.

Para conceber $\lambda$ suponha uma função $\psi:[0,+\infty[x[0,+\infty[\rightarrow R$ estritamente positiva e estritamente crescente em cada uma das variáveis e $\lambda(i, j)=\psi\left(w_{j}, d_{i j}\right)$, em que $w=\left(w_{i}: 1 \leq i \leq n\right): w_{i}>0$ é o peso atribuído à cidade $i$. A função usada foi $\psi(y, z)=(y+\varepsilon)(z+\delta), \varepsilon, \delta>0 . \lambda: C x C \rightarrow R$, é uma função estritamente positiva, quanto menor $\lambda(i, j)$, mais fácil ou barato é acessar o hospital localizado em $i$ baseando-se na localização $j$.

A matriz $\left(d_{i j}\right)$ foi obtida com base nas distâncias entre os municípios, valendo-se de dados geoprocessados. A distância média percorrida pelos usuários para se deslocar em cada zona (assumida não nula) foi calculada usando-se o valor esperado da distância média de deslocamento dos usuários. O valor esperado da distância usado para compor a diagonal principal da matriz é dado por 15:

$$
E(D)=(1,3)(0,38) \sqrt{A_{0}}
$$

Em que $A_{0}$ é a área total da zona, 1,3 é um coeficiente de correção e 0,38 é o valor da constante de proporcionalidade. $\mathrm{O}$ tempo de viagem foi obtido por meio da matriz de distâncias, considerando-se uma velocidade média de $60 \mathrm{~km} / \mathrm{h}$ entre municípios e $45 \mathrm{~km} / \mathrm{h}$ para deslocamentos internos na zona. Um peso foi atribuído a cada nó de demanda. Para fins de simulação, considerou-se como peso $10 \%$ da população do município (Instituto Brasileiro de Geografia e Estatística. http://www.cidades.ibge. gov.br/xtras/perfil.php?lang=\&codmun=421480\&search=santa-catarina $\mid$ rio-do-sul, acessado em 17/Jul/2014). 


\section{Resultados}

Para a validação do método proposto escolheu-se uma região razoavelmente homogênea quanto à distribuição de renda, densidade demográfica, perfil epidemiológico e qualidade de vias de acesso. Regiões que gozem de homogeneidade são preferíveis porque variáveis desconhecidas ou desprezadas tendem a não se tornar relevantes, gerando resultados mais confiáveis. No interior de Santa Catarina pode-se vislumbrar a Região de Saúde do Alto Vale do Itajaí como um exemplo que se aproxima desse ideal, principalmente porque o grau de incerteza quanto ao esforço despendido para o deslocamento da população é baixo, de forma a servir satisfatoriamente aos testes.

A Região de Saúde do Alto Vale do Itajaí pertence à macrorregião de Saúde do Vale do Itajaí, composta por 28 municípios, com uma população de referência de 579.946 habitantes 17 . Os municípios que a compõem são: Agrolândia, Agronômica, Atalanta, Aurora, Braço do Trombudo, Chapadão do Lageado, Dona Emma, Ibirama, Imbuia, Ituporanga, José Boiteux, Laurentino, Lontras, Mirim Doce, Petrolândia, Pouso Redondo, Presidente Getúlio, Presidente Nereu, Rio do Campo, Rio do Oeste, Rio do Sul, Salete, Santa Terezinha, Taió, Trombudo Central, Vidal Ramos, Vitor Meireles e Witmarsum. Rio do Sul é o município mais populoso e conta com 61.198 habitantes, segundo o Censo Demográfico de 2010 (Instituto Brasileiro de Geografia e Estatística. http://www.cidades.ibge.gov.br/xtras/perfil. php?lang $=\&$ codmun $=421480 \&$ search $=$ santa-catarina|rio-do-sul, acessado em 17/Jul/2014).

Para efeitos de modelagem, a Região de Saúde do Alto Vale do Itajaí foi discretizada numa rede, composta por zonas interligadas por vias de acesso. A região desfruta de municípios com estradas entre as cidades de qualidade razoável, independentes de condições climáticas que não sejam extremas. Uma zona é representada por um nó (ponto de referência central) e é candidata a abrigar uma unidade hospitalar. Cada município constitui uma zona, com exceção de Rio do Sul, que por ter mais de 50 mil habitantes foi dividido em duas. Segundo dados da Secretaria de Saúde da Estado de Santa Catarina (SES/SC), a área analisada conta com 15 hospitais que prestam atendimento pelo Sistema Único de Saúde (SUS). A distribuição geográfica atual das unidades hospitalares existentes na região base deste estudo, bem como a localização dos municípios pertencentes à Região de Saúde do Alto Vale do Itajaí e a posição ocupada pela região em Santa Catarina podem ser observadas nas Figuras 1 e 2.

Três cenários foram considerados:

(a) Cenário 1: avaliação da configuração atual do sistema em uso, em que aplica-se o indicador de acessibilidade em cada zona até um conjunto de hospitais mais próximos;

(b) Cenário 2: resolução do problema de localização - solução ótima segundo o modelo considerando-se apenas eficiência - e posteriormente cálculo do indicador de acessibilidade para cada zona até o conjunto de hospitais; e

(c) Cenário 3: resolução do problema de localização com inserção do indicador de acessibilidade. Em todos os casos foram utilizados os cinco hospitais mais próximos para avaliar a acessibilidade.

A coleta de dados foi realizada no decorrer de 2013 e no primeiro semestre de 2014. Informações como a população e a área dos municípios foram obtidas por meio de dados do Censo Demográfico de 2010. A configuração atual das unidades hospitalares de Santa Catarina foi adquirida na SES/SC, considerando-se os hospitais em funcionamento no ano de 2014 e que atendem pelo SUS.

Levando-se em conta a distribuição atual dos hospitais na Região de Saúde do Alto Vale do Itajaí, o indicador de acessibilidade apresentado na Equação 1 foi calculado, com a finalidade de analisar a situação real e compará-la com os resultados dos cenários simulados. A Tabela 1 mostra os valores máximos e mínimos do indicador de acessibilidade, bem como o valor de $\beta$, para a configuração atual (cenário 1) e para a configuração otimizada (cenário 2), em que inicialmente o problema de localização foi resolvido e depois o indicador de acessibilidade foi calculado.

A Figura 1 mostra a distribuição dos hospitais na Região do Alto Vale do Itajaí que foi resultado da solução do problema de localização (Equações de 3 a 6) apresentado neste trabalho e a distribuição atual para as unidades hospitalares. Visualmente é possível observar que os recursos estão espacialmente melhor distribuídos dado o cenário otimizado do que na configuração atual.

O cenário 2 é a solução mais eficiente encontrada pelo modelo, que desconsidera qualquer restrição relativa à acessibilidade. Essa solução apresentou uma diferença maior entre os valores máximos e mínimos de acessibilidade, porém a análise visual das unidades permite observar que existe uma homogeneidade maior na distribuição dessas instalações. 


\section{Figura 1}

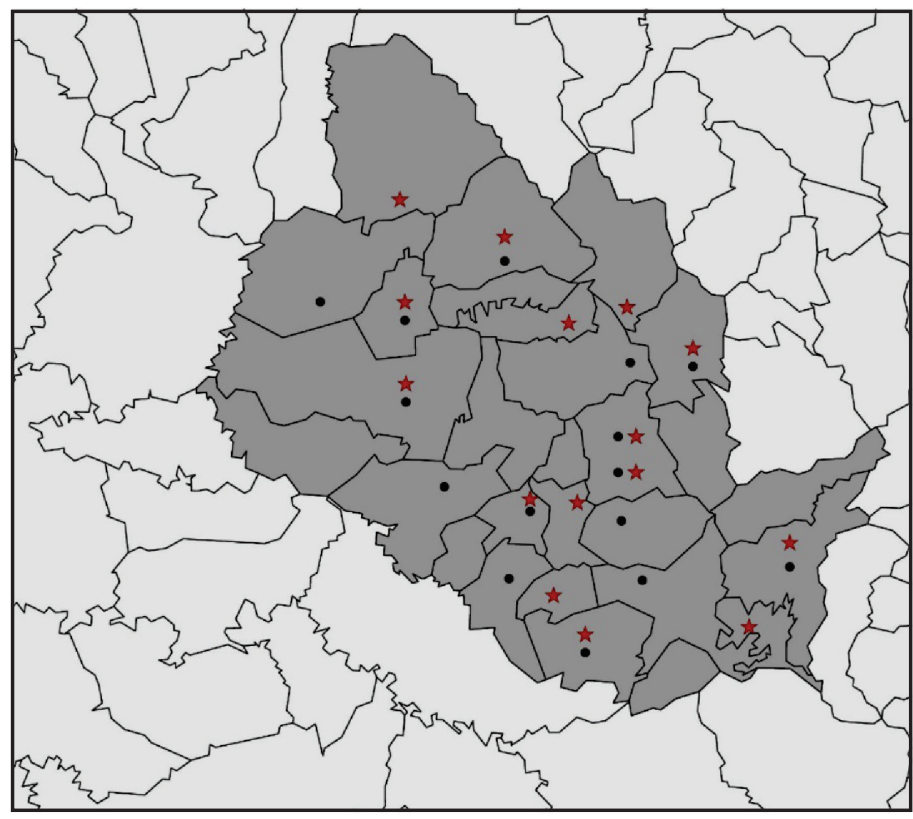

- Hospitais - cenário 1 (atual)

$\star$ Hospitais - cenário 2 (otimizado)

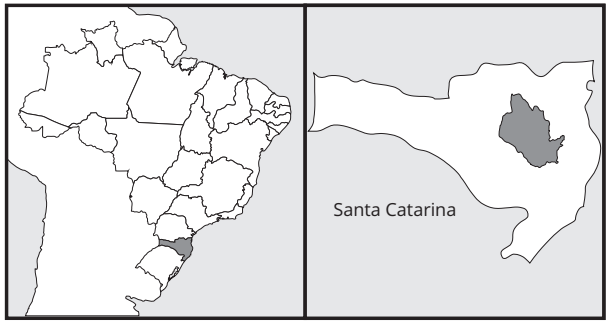

A Tabela 2 mostra os valores máximos e mínimos do indicador de acessibilidade, bem como o valor de $\beta$ solicitado e o valor encontrado para o cenário 3, em que o problema de localização foi resolvido com a inserção do indicador de acessibilidade apresentado na Equação 1 usando-se a Equação 2.

A inserção do indicador de acessibilidade no modelo de localização (cenário 3) permitiu obter a menor diferença entre os valores máximos e mínimos da acessibilidade e ainda assim manter a homegeneidade apresentada no cenário 2 (solução ótima segundo o modelo), o que pode ser observado na Figura 2.

$\mathrm{Na}$ Tabela 3, as faixas estabelecidas para cada nível de acessibilidade e o percentual das 29 zonas que se encontram em cada uma delas para a análise dos três cenários são apresentados. Como os valores do indicador são baixos, entre 0,31 e 0,80, cinco faixas foram estabelecidas. Os dados estão em ordem decrescente em razão de quanto maior o valor, melhor a acessibilidade.

Ao analisar os percentuais apresentados na Tabela 3, nota-se que o cenário 2 apresenta mais de $65 \%$ das zonas com valores mais elevados para o indicador de acessibilidade. A solução do problema de localização minimiza o tempo de viagem gasto pelos usuários entre as zonas, o que reflete um percentual maior de usuários nas faixas mais altas de acessibilidade aos serviços. Por outro lado, o cenário 


\section{Figura 2}

Distribuição dos hospitais dada a configuração atual e a configuração ótima com indicador de acessibilidade incorporado ao modelo de localização. Região de Saúde do Alto Vale do Itajaí, Santa Catarina, Brasil.

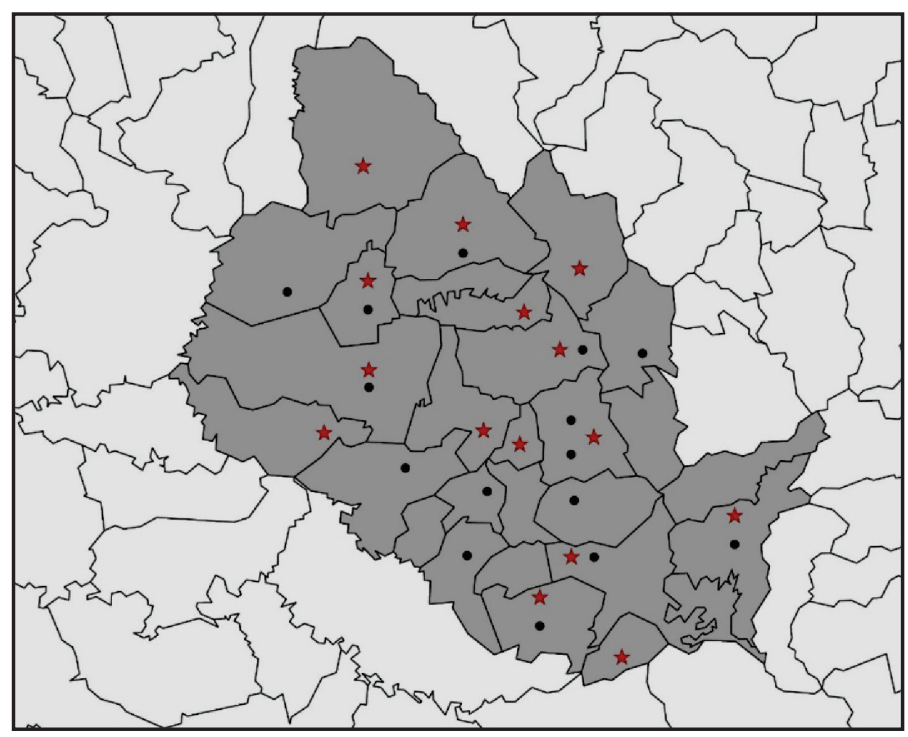

- Hospitais - cenário 1 (atual)

$\star$ Hospitais - cenário 2 (inserção de restrição)

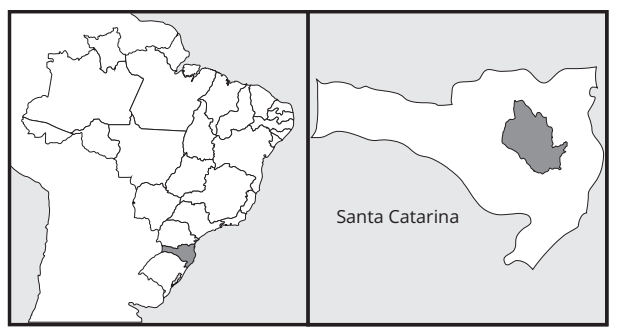

2 foi o que apresentou a menor equidade no acesso. Ao investigar o cenário 3 , com a melhor equidade entre os usuários em relação ao acesso aos serviços de saúde, percebe-se que não existem zonas nas faixas menos e mais elevadas de acessibilidade (entre 0,30 e 0,40; e entre 0,70 e 0,80). Os usuários estão alocados, na grande maioria, nas faixas intermediárias, ou seja, entre 0,50 e 0,70, indicando a redução das desigualdades, objetivo a ser alcançado quando se tem a equidade como princípio.

Conhecidos os valores do indicador de acessibilidade para o cenário atual e para os cenários simulados, em que a localização foi obtida por meio da solução do problema, sem e com a inserção do indicador de acessibilidade, é possível avaliar o impacto da intervenção sobre a acessibilidade dos usuários e os ganhos para o sistema de saúde em termos de equidade, segundo o cálculo da relação entre o indicador de acessibilidade dos usuários de cada zona $i$ na situação atual e da acessibilidade dos usuários da mesma sub-região nos dois cenários, por meio da expressão 18 :

$$
R A_{i}=\frac{A_{i}}{A_{i}^{s}}
$$

Em que $A_{i}$ é a acessibilidade dos usuários da zona para a situação atual, $A_{i} s$ é a acessibilidade dos usuários da zona $i$, considerando-se cada um dos dois cenários simulados e $R A_{i}$ é a relação entre a 
Tabela 1

Indicador de acessibilidade dada a configuração atual e a configuração ótima das unidades hospitalares existentes na Região de Saúde do Alto Vale do Itajaí, Santa Catarina, Brasil.

\begin{tabular}{lcc}
\hline & Cenário 1 & Cenário 2 \\
\hline Valor máximo de $A_{i}$ & 0,7436 & 0,7091 \\
Valor mínimo de $A_{i}$ & 0,4857 & 0,3975 \\
Valor de $\beta$ & 0,3468 & 0,4393 \\
\hline
\end{tabular}

Tabela 2

Indicador de acessibilidade obtido do problema de localização.

\begin{tabular}{lllllll}
\hline & $\boldsymbol{\beta}=\mathbf{0 , 1}$ & $\boldsymbol{\beta}=\mathbf{0 , 2}$ & $\boldsymbol{\beta}=\mathbf{0 , 3}$ & $\boldsymbol{\beta}=\mathbf{0 , 4}$ & $\boldsymbol{\beta}=\mathbf{0 , 5}$ & $\boldsymbol{\beta}=\mathbf{0 , 6}$ \\
\hline Valor máximo de $A_{i}$ & 0,6739 & 0,6779 & 0,6733 & 0,6710 & 0,7068 & 0,6999 \\
Valor mínimo de $A_{i}$ & 0,4808 & 0,4692 & 0,4529 & 0,4660 & 0,4484 & 0,4103 \\
Valor calculado de $\beta$ & 0,2865 & 0,3079 & 0,3273 & 0,3055 & 0,3656 & 0,4138 \\
\hline
\end{tabular}

\section{Tabela 3}

Percentual das zonas em cada faixa do nível de acessibilidade para os três cenários estabelecidos.

\begin{tabular}{lccc}
\hline Faixa do nível de acessibilidade & Cenário 1 (\%) & Cenário 2 (\%) & Cenário 3 (\%) \\
\hline $0,70<A_{i} \leq 0,80$ & 6,90 & 20,69 & 0,00 \\
$0,60<A_{i} \leq 0,70$ & 34,48 & 44,83 & 41,38 \\
$0,50<A_{i} \leq 0,60$ & 48,28 & 31,03 & 55,17 \\
$0,30<A_{i} \leq 0,50$ & 6,90 & 3,45 & 3,45 \\
$0,30<A_{i} \leq 0,40$ & 3,45 & 0,00 & 0,00 \\
\hline
\end{tabular}

acessibilidade dos usuários de cada zona na configuração atual e a acessibilidade dos usuários da mesma zona em cada um dos cenários simulados (problema de localização sem e com a inserção do indicador de acessibilidade).

Valores de $R A_{i}>1$ denotam que a acessibilidade dos usuários da sub-região no cenário simulado é melhor do que a acessibilidade dos mesmos usuários na situação atual, apresentando ganho em termos de equidade ao sistema de saúde em tela. Das 29 zonas consideradas, cinco apresentaram $R A_{i}<1$ para o cenário 2 , e apenas três apresentaram $R A_{i}<1$ para o cenário 3 , ou seja, não registraram melhorias em termos de equidade ao relacionar a situação atual com a configuração ótima calculada.

Analisar a relação existente entre os cenários simulados e o cenário atual é importante devido à pequena diferença apresentada entre os valores obtidos para a acessibilidade nos três cenários. Apesar de o valor de $\beta$ ser maior para o cenário 2 do que o cenário atual, a razão entre eles demonstra que apenas 5 zonas não apresentaram melhorias. E na comparação entre o cenário 3 e o atual, somente 3 zonas não registraram seu indicador melhorado. Destaca-se, com isso, que o modelo de localização apresentado e a inserção do indicador trazem melhorias para o sistema em termos de equidade.

Outra forma de medir a equidade é avaliando o grau de dispersão dos valores do indicador $A_{i} \mathrm{em}$ torno do valor médio. Para isso, medidas como o desvio médio e o desvio padrão são utilizadas 19. 
Quanto mais próximos da média, mais equitativo é o sistema. Os valores para o desvio médio e o desvio padrão para os três cenários foram, respectivamente, 0,0498 e 0,2682 para o cenário 1; 0,0642 e 0,3456 para o cenário 2; e 0,0461 e 0,2485 para o cenário 3 .

Os resultados obtidos para as medidas de equidade apontam para uma melhoria na equidade do cenário 3 (otimização com inserção do indicador de acessibilidade) em relação ao cenário 1 (atual), indicando o benefício da incorporação do indicador no modelo de localização. Já para o cenário 2 (ótimo), não foram apresentados benefícios em termos de equidade, pois o objetivo do problema de otimização é minimizar a distância média percorrida pelos usuários. Os resultados são coerentes, tendo em vista que o valor obtido para $\beta$ foi maior no cenário 2 do que nos demais, e que o cenário 3 apresentou valor menor em relação ao cenário 1 (Tabelas 1 e 2).

A solução do problema foi encontrada com o código implementado em MatLab R2008a (The MathWorks, Natick, Estados Unidos), usando-se as funções fminunc e fminsearch da toolbox de otimização e um microcomputador com processador Intel Core I5 com 4Gb de memória RAM e sistema operacional Windows 8 (Microsoft Corp., Estados Unidos).

\section{Discussão}

O equilíbrio entre eficiência e equidade é delicado num sistema de saúde 20 . Otimizar eficiência locacional significa almejar que o máximo possível de usuários seja servido para um dado orçamento público, com o mínimo de custo para os usuários como um todo. Em contrapartida, otimizar equidade significa distribuir o serviço de forma a que todos os usuários comprometam um esforço compatível com a sua necessidade para alcançá-lo 21. Pode-se, então, inferir que eficiência busca maximizar a distribuição dos serviços entre a população e que equidade busca maximizar a distribuição do efeito dos serviços entre a população. Como corolário, há uma grande possibilidade de que as funções matemáticas, que têm como imagem eficiência e equidade de um sistema, tenham valores máximos em posições distintas, e que para melhorar um se torne necessário prejudicar o outro. O modelo apresentado contempla esse trade-off entre equidade e eficiência, utilizando um indicador que orienta a função objetivo a alcançar uma solução com um nível de acessibilidade esperado, considerado plausível. Removendo-se o indicador otimiza-se a eficiência. Em suma, o modelo apresentado não otimiza a equidade, e sim busca um resultado que seja próximo do mais eficiente, porém com maior grau de equidade. Diminuir a desigualdade na acessibilidade aos cuidados de saúde identifica lacunas que devem ser fechadas, ou pelo menos reduzidas 22.

Vale salientar que trabalhos que analisam a acessibilidade em sistemas de saúde são bastante presentes na literatura. Dentre eles vale salientar Wang 22, que apresenta uma revisão metodológica que envolve medidas, otimização e o impacto da análise da acessibilidade em sistemas de saúde. Culyer \& Wagstaff 23 exploram diferentes definições de equidade na atenção à saúde. Moonay et al. 24 analisam as definições sobre equidade apresentadas em diversos artigos e indicam qual objetivo consideram mais importante em termos de políticas de saúde. Ferreira \& Raffo 13 realizam uma abrangente revisão da literatura sobre acessibilidade, principalmente no que tange à sua taxonomia e na categoria de modelos. Os autores distinguem quatro formas de medir acessibilidade espacialmente: (i) proporção provedor-população; (ii) distância até o provedor mais próximo; (iii) distância média até um conjunto de provedores; e (iv) modelos gravitacionais de influência espacial.

Os modelos gravitacionais combinam acessibilidade e disponibilidade de serviço, sendo diretamente proporcional à atratividade e inversamente à distância da moradia do usuário e do provedor de serviço. Guagliardo 5 considera que as medidas obtidas por modelos gravitacionais são as mais realistas, seja com geografias urbanas ou rurais. A atratividade de uma instalação de saúde pode ser arbitrada sob diferentes aspectos, como número de especialidades disponíveis, padrão de atendimento, renome etc. No presente trabalho, considerou-se atratividade como o número de leitos à disposição, principalmente por ser uma medida confiável e que reflete o porte do sistema, o que por fim tende a ter correlação com outros parâmetros que fazem com que as pessoas procurem, ou deem preferência, a uma determinada instalação.

O modelo proposto, portanto, não visa a obter a solução de menor custo social para a localização do sistema de saúde, mas de obter um resultado não muito distante desta solução, que carregue con- 
sigo uma melhoria significativa de acessibilidade. Pode-se dizer, logo, que algumas ineficiências são admitidas no modelo visando a dar maior acessibilidade ao conjunto de usuários, fazendo com que o alcance do serviço aumente sem custos adicionados que tornem o sistema inviável.

A aplicação da metodologia utilizada neste estudo, para a localização de unidades prestadoras de serviços de saúde, é mais uma ferramenta a estar disponível para o auxílio à decisão do gestor, e possibilita um olhar quantitativo quanto à distribuição das unidades de atendimento e ao alcance da rede a populações menos favorecidas geograficamente. Estudos como este permitem simulações que podem dar origem a um planejamento justificado, seja para ampliar uma rede acompanhando o crescimento demográfico de uma região, seja para reduzir um sistema ineficiente, evitando ações improvisadas. O modelo apresentado pode ter ainda sua utilização estendida a outros tipos de serviços públicos, como escolas e centros de atendimento ao cidadão, permitindo medidas que aumentem a equidade do sistema e diminuam desperdícios por baixa procura às instalações.

Foi apresentado, no trabalho, um critério para calcular atratividade e acessibilidade. O modelo, no entanto, é versátil, e permite decidir por outras variáveis que sejam relevantes num dado contexto. Quando se considera apenas o problema de localização, mais zonas apresentaram valores melhores em termos de acessibilidade, porém a diferença entre os maiores e menores valores é maior. Já o problema de localização com a aplicação do indicador produz soluções que gozam de menor eficiência econômica, mas que são ainda bastante viáveis, e com disparidade reduzida em relação à acessibilidade dos serviços prestados.

\section{Colaboradores}

S. Cirino trabalhou na concepção e delineamento do artigo, coleta, análise e interpretação de dados, redação e revisão crítica. L. A. Gonçalves participou na análise, interpretação de dados, redação e revisão crítica. M. B. Gonçalves participou na concepção, delineamento e revisão crítica. E. S. Cursi participou da construção do modelo, elaboração do código de otimização e na revisão crítica.

\section{Agradecimentos}

Agradecimentos à Coordenação de Aperfeiçoamento de Pessoal de Nível Superior (Capes) pela concessão de bolsa de doutorado sanduíche à $\mathrm{S}$. Cirino (processo no 6283-13-6). Ao Conselho Nacional de Desenvolvimento Científico e Tecnológico (CNPq) pelo financiamento. $\mathrm{O}$ trabalho foi apresentado no XXVIII ANPET - Congresso Nacional de Pesquisa e Ensino em Transportes, Curitiba, Brasil, em 2014 (os autores declaram que o congresso é de uma área diferente da área de saúde coletiva). 


\section{Referências}

1. Souza MSPL. Acessibilidade geográfica aos serviços de saúde, retardo do diagnóstico e abandono dos casos de tuberculose [Tese de Doutorado]. Salvador: Instituto de Saúde Coletiva, Universidade Federal da Bahia; 2015.

2. Litman T. Evaluating accessibility for transport planning: measuring people's ability to reach desired goods and activities. http://www.vtpi. org/access.pdf (acessado em Fev/2017).

3. Davidson K. Accessibility and isolation in transport network evaluation. http:// transposition.com.au/research/1995Accessibility.pdf (acessado em Fev/2017).

4. Cirino S, Gonçalves LA, Gonçalves MB, Cursi ES, Coelho AS. Avaliação de acessibilidade geográfica em sistemas de saúde hierarquizados usando o modelo de p-medianas: aplicação em Santa Catarina, Brasil. Cad Saúde Pública 2016; 32:e00172614.

5. Guagliardo MF. Spatial accessibility of primary care: concepts, methods and challenges. Int J Health Geogr 2004; 3:3.

6. Souza CCA, Martins RS, Figueiredo L, Lemos MB. Indicador de acessibilidade para análise do desenvolvimento regional. Revista Econômica do Nordeste 2010; 41:581-98.

7. Engel RH, Weiller TH, Farão EMD, Barros IFO, Bordignon J, Luz EMF. Acessibilidade geográfica e organizacional: obstáculos entre usuários com tuberculose e os serviços de saúde. Rev Enferm UFSM 2013; 3:307-14.

8. Rahman S, Smith DK. Use of location-allocation models in health service development planning in developing nations. Eur J Oper Res 2000; 123:437-52.

9. Almeida LMW, Gonçalves MB. Determinação de índices de acessibilidade a serviços escolares. In: Anais do XI Congresso Pan-Americano de Engenharia de Trânsito e Transporte, Engenharia de Tráfego e Transportes. Gramado: Associação Nacional de Pesquisa e Ensino em Transportes; 2000. p. 453-65.

10. Cirino S. Modelo de p-medianas hierárquico e acessibilidade: análise dos hospitais públicos de Santa Catarina [Tese de Doutorado]. Florianópolis: Centro Tecnológico, Universidade Federal de Santa Catarina; 2016.

11. Silva Júnior ES, Medina MG, Pereira RAG, Fonseca ACF, Vilasbôas ALQ. Acessibilidade geográfica à atenção primária à saúde em distrito sanitário do Município de Salvador, Bahia. Rev Bras Saúde Matern Infant 2010; 10 Suppl 1:S49-60.
12. Pooler JA. The use of spatial separation in the measurement of transportation accessibility. Transp Res Part A Policy Pract 1995; 29:421-7.

13. Ferreira RV, Raffo JG. O uso dos sistemas de informação geográfica (SIG) no estudo da acessibilidade física aos serviços de saúde pela população rural: revisão da literatura. Revista Brasileira de Geografia Médica e da Saúde 2012; 8:177-89.

14. Pirie GH. Measuring accessibility: a review and proposal. Environ Plann A 1979;11:299312.

15. Jamtsho S, Corner RJ. Evaluation of spatial accessibility to primary healthcare using GIS. ISPRS Annals of the Photogrammetry, Remote Sensing and Spatial Information Sciences 2014; 2:79-86.

16. Cirino S, Gonçalves MB, Cursi ES. Indicador de acessibilidade em modelos de localização: análise dos hospitais públicos de Santa Catarina. In: Anais do XXVIII ANPET Congresso de Pesquisa e Ensino em Transportes. Curitiba: Associação Nacional de Pesquisa e Ensino em Transportes; 2014. p. 1-12.

17. Secretaria de Estado da Saúde. Plano Estadual de Saúde de Santa Catarina, 2012-2015. http://portalses.saude.sc.gov.br/index.php? option=com_docman\&task=doc_download \&gid=5771\&Itemid=251 (acessado em 01/Jul/ 2014).

18. Cirino S, Lima FS, Gonçalves MB. Spatial distribution of specialized cardiac care units in the state of Santa Catarina. Rev Saúde Pública 2014; 48:916-24.

19. Mulligan GF. Equality measures and facility location. Pap Reg Sci 1991; 4:345-65.

20. Truelove M. Measurement of spatial equity. Environ Plann C Gov Policy 1993; 11:19-34.

21. Braveman P, Gruskin S. Defining equity in health. J Epidemiol Community Health 2003; 57:254-8.

22. Wang F. Measurement, optimization, and impact of health care accessibility: a methodological review. Ann Assoc Am Geogr 2012; 102:1104-12.

23. Culyer AJ, Wagstaff A. Equity and equality in health and health care. J Health Econ 1993; 12:431-57.

24. Moonay G, Hall J, Donaldson C, Gerard K. Utilisation as a measure of equity: weighing heat? J Health Econ 1991; 10:475-80. 


\section{Abstract}

The study proposes a differentiated approach to the localization of public services (unlike methods focusing solely on locational efficiency in the distribution of such services), with a nonlinear model that incorporates an accessibility indicator and allows rejecting solutions in which accessibility fails to comply with acceptably established minimum parameters. The method aims to minimize the total time spent by a region's population to reach a public services network, while controlling the range between the highest and lowest accessibility to the services. The resulting solution is not as efficient as other models (e.g., $p$-median) in relation to total cost for the population as a whole to access the system, but it seeks to prevent the most distant areas from experiencing greater difficulty due to their disproportional traveling time. The model was tested in a region in the hospital network of the State of Santa Catarina, Brazil, and the results show that incorporation of the indicator suggests improvement when compared to the current distribution of hospitals in that area. The proposed methodology can be a useful tool for planning balanced resource allocation during installation of health services for the population.

Nonlinear Dynamics; Health Services Accessibility; Health Services;

Health Planning

\section{Resumen}

Este trabajo propone un enfoque diferencial para la localización de unidades prestadoras de servicio público. A diferencia de métodos que se atienen únicamente a la eficiencia de localización para la distribución de unidades prestadoras de servicios públicos, se presenta un modelo no lineal que incorpora un indicador de accesibilidad en su seno, que permite rechazar soluciones cuya accesibilidad esté fuera de parámetros mínimos considerados como aceptables. El método tiene como objetivo minimizar el tiempo total invertido por parte de la población de una región, con el fin de alcanzar una red de prestación de servicios públicos, incluso controla la amplitud entre la región con la mayor y menor accesibilidad a la red de prestación de servicios. La solución que se obtiene no es tan eficiente, cuando se compara con otros modelos como pmedianas, en lo que se refiere al coste total gastado por la población como un todo para tener acceso al sistema, sin embargo, busca evitar que las áreas más apartadas tuvieran mayores dificultades, en virtud de la desproporción del tiempo de viaje necesario. El modelo fue probado en una región de la red hospitalaria del estado de Santa Catarina, Brasil, y los resultados muestran que la incorporación del indicador apunta a una mejoría, en relación con la coyuntura actual de la distribución de unidades hospitalarias. El uso de la metodología sugerida puede ser útil, como una herramienta que contribuye con apoyos, a la elaboración de una planificación que permita una distribución equilibrada de recursos, en lo referente a la instalación de unidades prestadoras de servicios a la población.

Dinámicas no Lineales; Accesibilidad a los Servicios de Salud; Servicios de Salud; Planificación en Salud
Recebido em 09/Nov/2015

Versão final reapresentada em 15/Ago/2017 Aprovado em 04/Set/2017 